Case Report

\title{
Posterior Microphthalmia, Peripheral Pigmentary Retinal Changes, Yellow Lesions, and Cleft Lip: A Case Report and Literature Review
}

\author{
Nasser G. Alsaedi $\mathbb{D}^{1,2}$ and Khalid Alrubaie $\mathbb{D}^{1}$ \\ ${ }^{1}$ King Khaled Eye Specialist Hospital, Riyadh, Saudi Arabia \\ ${ }^{2}$ King Abdullah Medical City, Makkah, Saudi Arabia \\ Correspondence should be addressed to Nasser G. Alsaedi; alsaaadi9@hotmail.com
}

Received 25 November 2018; Revised 16 April 2019; Accepted 5 May 2019; Published 19 May 2019

Academic Editor: Stephen G. Schwartz

Copyright (C) 2019 Nasser G. Alsaedi and Khalid Alrubaie. This is an open access article distributed under the Creative Commons Attribution License, which permits unrestricted use, distribution, and reproduction in any medium, provided the original work is properly cited.

\begin{abstract}
Purpose. Posterior microphthalmia is a sporadic or inherited developmental ocular anomaly that may occur isolated or in association with multiple ocular and systemic anomalies. This report documents a case of posterior microphthalmia with atypical presentation including white dots in the posterior pole in addition to systemic anomalies including facial defect that can represent an underlying genetic mutation. Method. Case report. Results. A 29-year-old male with high hyperopia and history of bilateral clear lens presented with pigmentary changes and white-yellow dots in the posterior pole in both eyes. Patient had a history of cleft lip repair. A complete ocular evaluation including A/B scan and optical coherence tomography confirmed the diagnosis of posterior microphthalmia with a retinitis pigmentosa like fundus and drusen deposits in the subretinal pigment epithelium. Conclusion. The white-yellow drusenoid deposits in the posterior pole in association with posterior microphthalmia are poorly documented in the literature. Cases of craniofacial developmental defects in association with posterior microphthalmia may represent a genetic defect.
\end{abstract}

\section{Introduction}

Posterior microphthalmia is a sporadic or inherited developmental ocular anomaly that may occur isolated or in association with multiple ocular and systemic anomalies. Ocular association including retinitis pigmentosa and optic disc drusen were first published by Buys et al. in 1999 [1]. In 2017, Plaza et al. documented white dots in the posterior pole as a new ocular finding associated with posterior microphthalmia [2]. Microphthalmia has been reported to be associated with multiple systemic anomalies including, mental retardation, craniofacial malformations (e.g., cleft lip/palate), and anomalies of the hands and feet and can be a finding in other congenital syndromes [3]. We document a rare case of posterior microphthalmia with other ocular and extraocular anomalies.

\section{Case Report}

A 29-year-old Saudi male presented to our institution (King Khaled Eye Specialist Hospital, KKESH) on 2007 seeking a refractive procedure. The patient had a history of cleft lip repair and had no systemic illness at presentation. The patient denied ocular trauma, ocular surgery or a family history of visual dysfunction. On ocular examination, the visual acuity in the right eye (OD) was 20/50 with a subjective cycloplegic refraction of $+15.25-0.75 \times 140^{\circ}$ and 20/30 in the left eye (OS) with a subjective cycloplegic refraction of $+15.00-0.50 \times 30^{\circ}$. The interpupillary distance was $63 \mathrm{~mm}$. Intraocular pressure in both eyes (OU) was $19 \mathrm{mmHg}$. Slit lamp examination was unremarkable OU. White-to-white corneal measurements were $12.2 \mathrm{~mm}$ OD and $11.5 \mathrm{~mm}$ OS as measured with the slit lamp. The steepest keratometry (K) was $48.3 \mathrm{D}$ at $31^{\circ} \mathrm{OD}$ and $48.8 \mathrm{D}$ at $116^{\circ} \mathrm{OS}$. The corneal thickness measurements were 


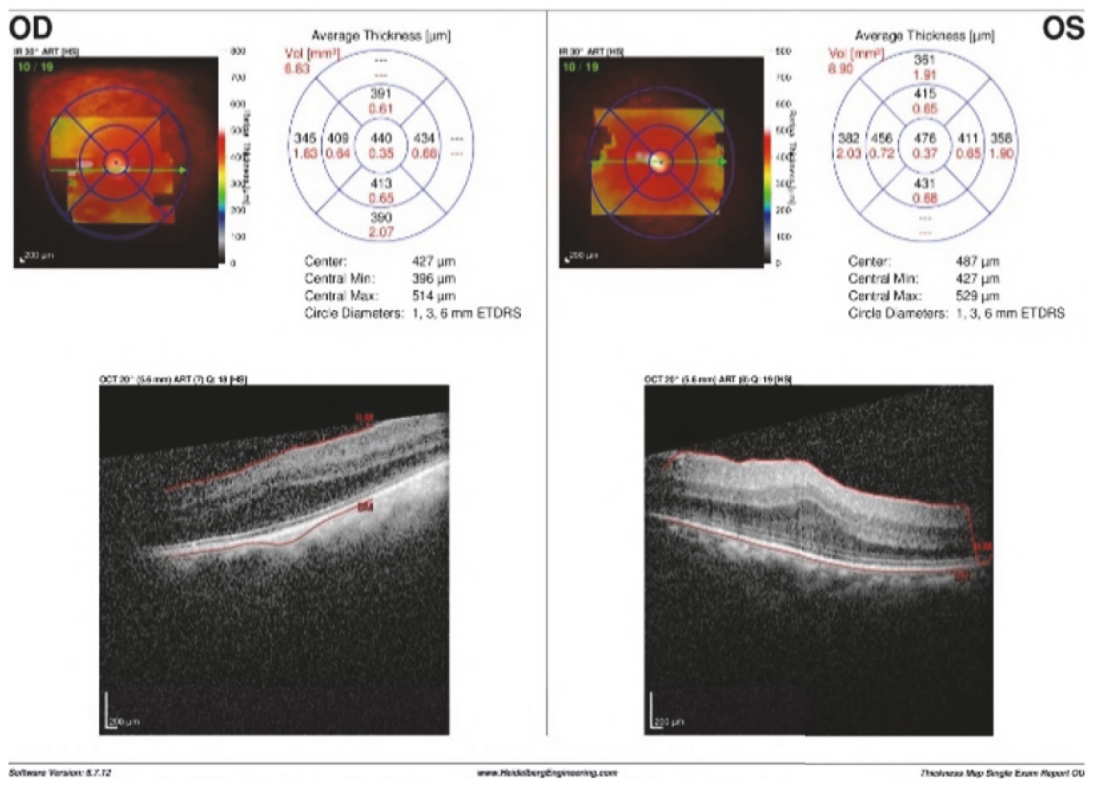

FIGURE 1: Spectral domain optical coherence tomography of both maculae and horizontal cut, depicting an inverted U-shaped papillomacular folds of the neurosensory retina, greater in the left eye with preservation of the majority of outer retinal layers.

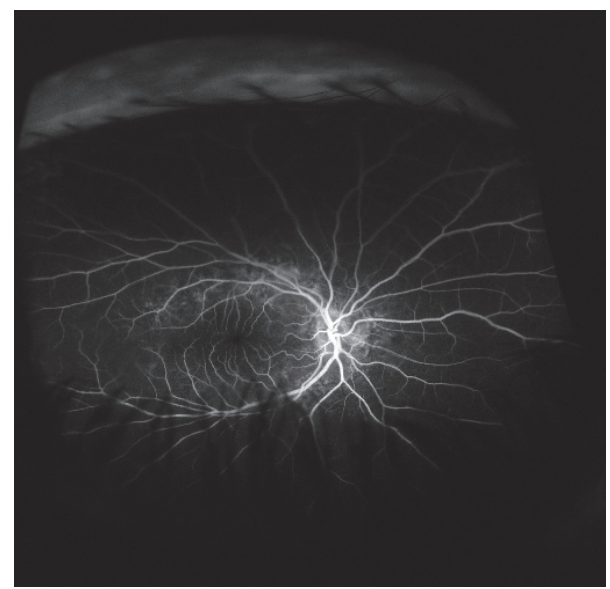

FIGURE 2: Fundus fluorescein angiography in later phases showing hyperfluorescent deposits in the posterior pole without leakage.

$512 \mu \mathrm{m}$ OD and $511 \mu \mathrm{m}$ OS. The biometric measurements as measured by (Orbscan IIz, Bausch and Lomb, Rochester, NY, USA) of the anterior chamber depth revealed $3.32 \mathrm{~mm}$ OD and $3.49 \mathrm{~mm}$ OS. Iris examination indicated patent peripheral YAG laser iridotomies bilaterally without correctopia OU Piggyback intraocular lenses were present OU.

Indirect ophthalmoscopy was remarkable for crowded optic discs and subretinal drusenoid yellow-white dots symmetrically distributed in the posterior pole OU. Bilaterally, the retinal blood vessels appeared normal, with no clinically obvious papillomacular folds and peripheral pigmentary bone spicule pigmentation.
Macular spectral-domain optical coherence tomography (SD-OCT) revealed inverted U-shaped papillomacular folds OU (Figure 1). Posterior microphthalmia was then suspected and A-scan axial length measurements were $16.40 \mathrm{~mm} \mathrm{OD}$ and $16.65 \mathrm{~mm}$ OS. Fluorescein angiography showed no optic nerve head staining or leakage. Staining of the yellowish subretinal drusenoids deposits was seen in the later frames OU (Figure 2). The diagnosis of posterior microphthalmia was confirmed with the given findings and no further intervention was recommended.

\section{Discussion}

Posterior microphthalmia is a rare developmental anomaly and is defined as a normal eye with multiple ocular findings that includes high hyperopia, normal or subnormal anterior segment structures, short axial length that may vary from $12.30 \mathrm{~mm}$ to $20.36 \mathrm{~mm}$, papillomacular folds, pseudo-papilledema, thick choroid, and sclera [4]. Posterior microphthalmia is considered a rare subtype of microphthalmia in which there is total axial length reduction with a normal or near normal cornea. Microphthalmos is defined as a structural defect with total axial length at least two standard deviations below age-matched controls or an anteroposterior diameter less than $20 \mathrm{~mm}$ in adults $[5,6]$. Microphthalmia is categorized generally into pure and complex, pure if there are no associated ocular defects and complex if there are other associated major ocular defects [7]. Our case report presented with ocular and systemic associations.

The association of microphthalmia with pigmentary retinal changes has been previously reported. In 1958, Hermann described 13 patients of 4 generations in a family that showed 
autosomal dominant transmission of microphthalmia with a variety of retinal pigmentary changes. Hermann also documented two cases reported by Catsch that had microphthalmia with retinitis pigmentosa [8].

In 1965, Franceschetti et al. described a nonconsanguineous family comprised of four individuals with microphthalmia, retinal degeneration, and dental anomalies. They postulated a probable autosomal recessive pattern of inheritance in this association (MIM 251700) [9]. In 1999, Buys et al. published the first combination of nanophthalmos, retinitis pigmentosa, and optic disc drusen that was observed in a 68year-old male and they proposed that a retinitis pigmentosalike presentation in such patients may be due to chronic choroidal effusion and serous retinal detachments [1]. These associations described by Buys et al. were subsequently shown to be associated with a frameshift mutation of MFRP (Membrane-type Frizzled-Related Protein) confirming the syndrome of posterior microphthalmos, retinitis pigmentosa, foveoschisis, and optic disc drusen [10]. Interestingly, the association of optic disc drusen with retinitis pigmentosa is well known in the literature. Grover et al. reviewed 262 patients with retinitis pigmentosa and found that $9.2 \%$ had optic disc drusen [11]. Another ocular finding in our case is the white/yellow dots in the posterior pole. These white dots in the posterior pole in association with posterior microphthalmia are similar to a case first reported by Plaza et al. in 2017 with posterior microphthalmia, retinitis pigmentosa, optic disc drusen syndrome, and additional findings of yellow macular dots that were not included as criteria for this syndrome [2].

Ayala-Ramires et al. described the criteria for the syndrome as follows: posterior microphthalmos with anteroposterior diameter between $13 \mathrm{~mm}$ and $18.5 \mathrm{~mm}$; normal corneal and anterior chamber diameters; high hyperopia, between +8.00 and $+25.00 \mathrm{D}$; optic nerve head drusen with campimetric defects similar to those caused by simple chronic glaucoma; retinal dystrophy compatible with pigment retinosis confirmed by electroretinogram in association with foveoschisis, cystoid macular edema, or foveolar thickening [12]. The yellow dots reported by Plaza et al. were located at the level of retinal pigmented epithelium and the fluorescein angiography indicated a broad window defect with hyperfluorescence of these dots in the later phases [2]. These yellow dots behave like drusen and we postulate that the association of this syndrome with drusen can be found in the disc or the retina.

Papillomacular fold is an ocular finding in posterior microphthalmia and is considered one of the causes of reduced vision. It is confined only to the neurosensory layer sparing the retinal pigment epithelium, choroid, and sclera [13]. It is important for the ophthalmologist to be aware of this finding as this can be confused or mistaken as cystoid macular edema [14]. Additionally, we recommend axial length measurements in patients with hyperopia over $+8.00 \mathrm{D}$ and similar posterior segment findings. The SD-OCT finding in our case indicates absence of the normal foveal pit or fovea plana that is also associated with microphthalmos, ocular albinism, retinopathy of prematurity, and aniridia $[15,16]$. The term fovea plana instead of foveal hypoplasia
TABle 1: A Practical Classification of Microphthalmia/Coloboma.

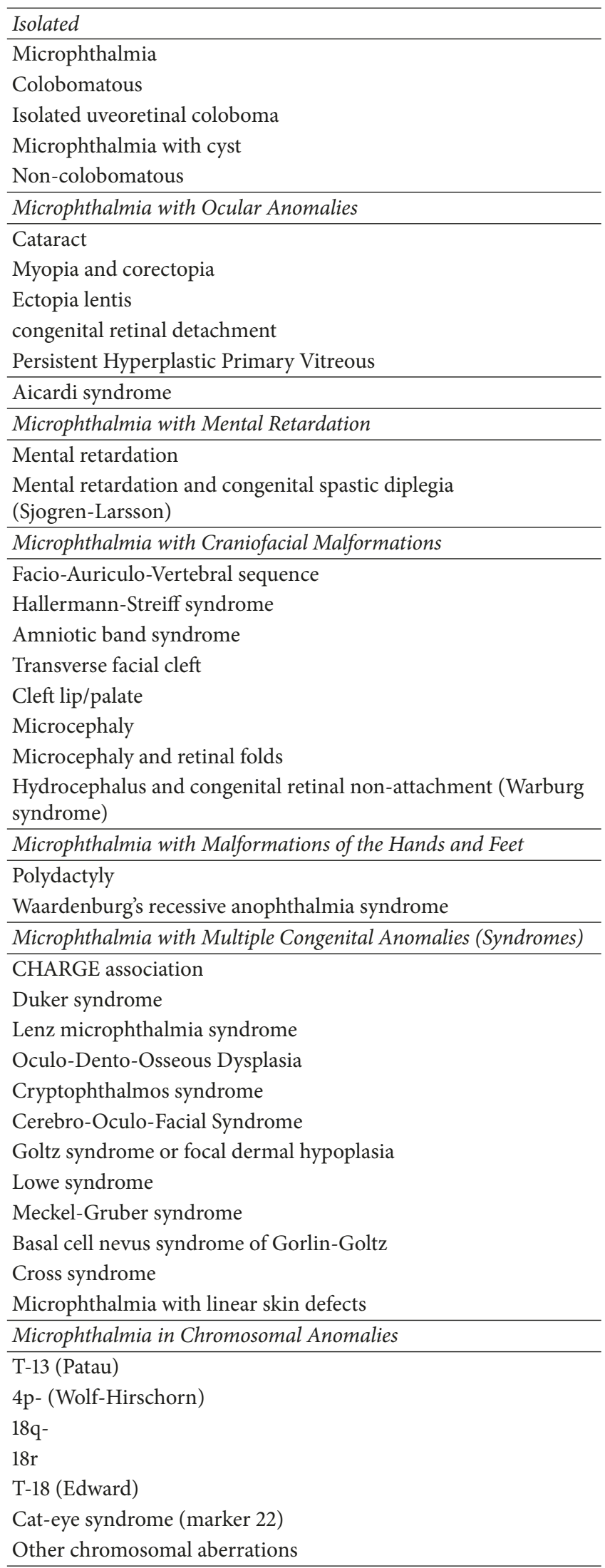


TABle 1: Continued.

\begin{tabular}{l}
\hline Microphthalmia and Intrauterine Insults \\
\hline Maternal drug intake: thalidomide, alcohol, isotretinoin, others \\
Maternal vitamin A deficiency \\
Maternal fever or radiation exposure \\
Maternal uncontrolled phenylketonuria \\
Intrauterine infections: CMV, EBV, Varicella, Herpes simplex, \\
Rubella, Toxoplasmosis \\
\hline
\end{tabular}

Adopted from [3].

was suggested by Marmor when the cone is preserved both anatomically and functionally despite the absence of the foveal pit [17]. Fovea plana can be found in normal eyes and up to a $3 \%$ incidence had been reported in children with clinically normal eyes [18].

The extraocular manifestation of patients with microphthalmia spectrum includes multisystemic malformations of the heart, renal, face, and central nervous system [19]. A systemic work-up should be performed in patients with multiple malformations and a chromosomal microarray study or exome sequencing may be required in those with multiple malformations (Table 1) [3].

\section{Conclusion}

Posterior microphthalmia should be suspected in cases with high hyperopia. The occurrence of posterior microphthalmia with other ocular and systemic associations has been reported in the literature. The white-yellow dots in the posterior pole in posterior microphthalmia are an atypical finding and poorly described in the literature. Cases of craniofacial developmental defects in association with ocular anomalies including posterior microphthalmia may represent genetic/chromosomal defects. We recommend evaluating such cases to establish a distinct pattern of inheritance.

\section{Conflicts of Interest}

In relation to this case report, the authors declare that there are no conflicts of interest.

\section{References}

[1] Y. M. Buys and C. J. Pavlin, "Retinitis pigmentosa, nanophthalmos, and optic disc drusen: A case report," Ophthalmology, vol. 106, no. 3, pp. 619-622, 1999.

[2] P. Plaza, O. Iturralde, and C. Abascal, "Posterior microphthalmos, retinitis pigmentosa and optic disc drusen with white dots. A case report," Archivos de la Sociedad Espanola de Oftalmologia, vol. 92, no. 11, pp. 555-558, 2017.

[3] O. Arif, M. D. Khan, and M. D. Elias Traboulsi, "Microphthalmia, anophthalmia, coloboma and nanophthalmos (includes Charge association)," 2016, https://www.aao.org.

[4] S. R. Nowilaty, A. O. Khan, M. A. Aldahmesh, K. F. Tabbara, A. Al-Amri, and F. S. Alkuraya, "Biometric and molecular characterization of clinically diagnosed posterior microphthalmos,"
American Journal of Ophthalmology, vol. 155, no. 2, pp. 361-372, 2013.

[5] R. Ayala-Ramirez, F. Graue-Wiechers, V. Robredo, M. AmatoAlmanza, I. Horta-Diez, and J. C. Zenteno, "A new autosomal recessive syndrome consisting of pasterior microphthalmos, retinitis pigmentosa, foveoschisis, and optic disc drusen is caused by a MFRP gene mutation," Molecular Vision, vol. 12, pp. 1483-1489, 2006.

[6] M. J. Elder, "Aetiology of severe visual impairment and blindness in microphthalmos," British Journal of Ophthalmology, vol. 78, no. 5, pp. 332-334, 1994.

[7] E. I. Traboulsi, "Colobomatou smicrophthalmia, anophthalmia, and associated malformation syndromes," in Genetic Diseases of the Eye, E. I. Traboulsi, Ed., pp. 51-80, Oxford University Press, New York, NY, USA, 1998.

[8] P. Hermann, "Le syndrome microphtalmie-rétinite pigmentaireglaucome," Archives of Ophthalmology, vol. 18, pp. 17-24, 1958.

[9] A. Franceschetti and H. Gernet, "Ultrasonics diagnosis of microphthalmia without microcornea, with macrophakia, high hypermetropia associated with tapeto-retinal degeneration, a glaucomatous predisposition and dental anomalies (new familial syndrome)," Archives d'Ophtalmologie et Revue Générale d'Ophtalmologie, vol. 25, pp. 105-116, 1965.

[10] J. Crespi, J. A. Buil, and F. Bassaganyas, "A novel mutation confirms MFRP as the gene causing the syndrome of nanophthalmos-renititis pigmentosa-foveoschisis-optic disk drusen," American Journal of Ophthalmology, vol. 146, no. 2, pp. 323-328, 2008.

[11] S. Grover, G. A. Fishman, and J. Brown Jr., "Frequency of optic disc or parapapillary nerve fiber layer drusen in retinitis pigmentosa," Ophthalmology, vol. 104, no. 2, pp. 295-298, 1997.

[12] R. Ayala-Ramirez, F. Graue-Wiechers, V. Roberdo, M. AmatoAlmanza, I. Horta-Diez, and J. C. Zenteno, "A new autosomalrecessive syndrome consisting of posterior microphthalmos,retinitis pigmentosa, foveoschisis, and optic disc drusen iscaused by a MFRP gene mutation," Molecular Vision, vol. 4, pp. 1483-1489, 2006.

[13] T. E. Jackson, Y. C. Yang, and G. A. Shun-Shin, "Spectral domain optical coherence tomography findings in retinal folds associated with posterior microphthalmos," Journal of American Association for Pediatric Ophthalmology and Strabismus, vol. 16, no. 4, pp. 389-391, 2012.

[14] A. A. Albar, S. R. Nowilaty, and N. G. Ghazi, "Posterior microphthalmos and papillomacular fold-associated cystic changes misdiagnosed as cystoid macular edema following cataract extraction," Clinical Ophthalmology, vol. 9, pp. 73-76, 2015.

[15] A. Vincent, V. Kemmanu, R. Shetty, V. Anandula, B. Madhavarao, and B. Shetty, "Variable expressivity of ocular associations of foveal hypoplasia in a family," Eye, vol. 23, no. 8, pp. 1735-1739, 2009.

[16] V. M. Villegas, S. G. Schwartz, T. D. Hamet, C. A. McKeown, H. Capó, and H. W. Flynn, "Variable clinical profile of fovea plana in normal children," Ophthalmic Surgery, Lasers and Imaging Retina, vol. 49, no. 4, pp. 251-257, 2018.

[17] M. F. Marmor, S. S. Choi, R. J. Zawadzki, and J. S. Werner, "Visual insignificance of the foveal pit: Reassessment of foveal hypoplasia as fovea plana," JAMA Ophtalmology, vol. 126, no. 7, pp. 907-913, 2008.

[18] S. Noval, S. F. Freedman, S. Asrani, and M. A. El-Dairi, "Incidence of fovea plana in normal children," Journal of American 
Association for Pediatric Ophthalmology and Strabismus, vol. 18, no. 5, pp. 471-475, 2014.

[19] E. Raveh, B. C. Papsin, and V. Forte, "Branchio-oculo-facial syndrome," International Journal of Pediatric Otorhinolaryngology, vol. 53, no. 2, pp. 149-156, 2000. 


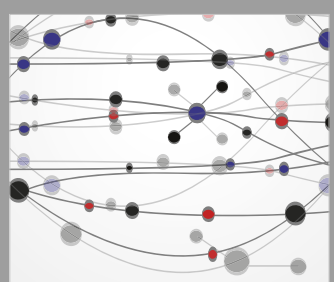

The Scientific World Journal
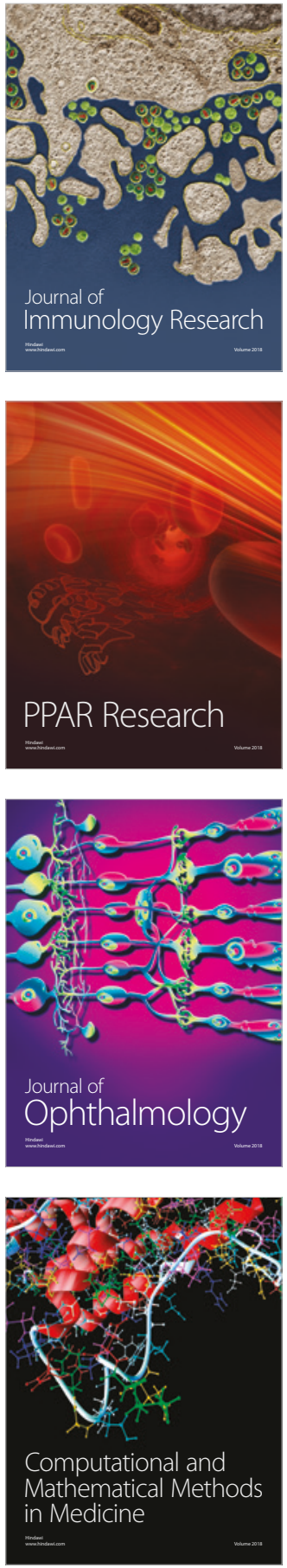

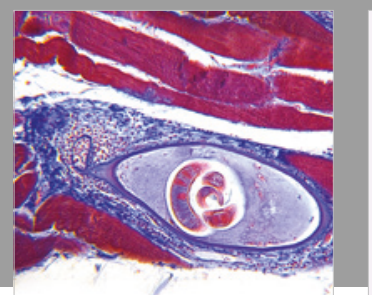

Gastroenterology Research and Practice

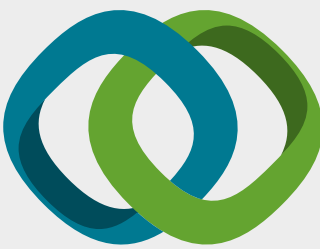

\section{Hindawi}

Submit your manuscripts at

www.hindawi.com
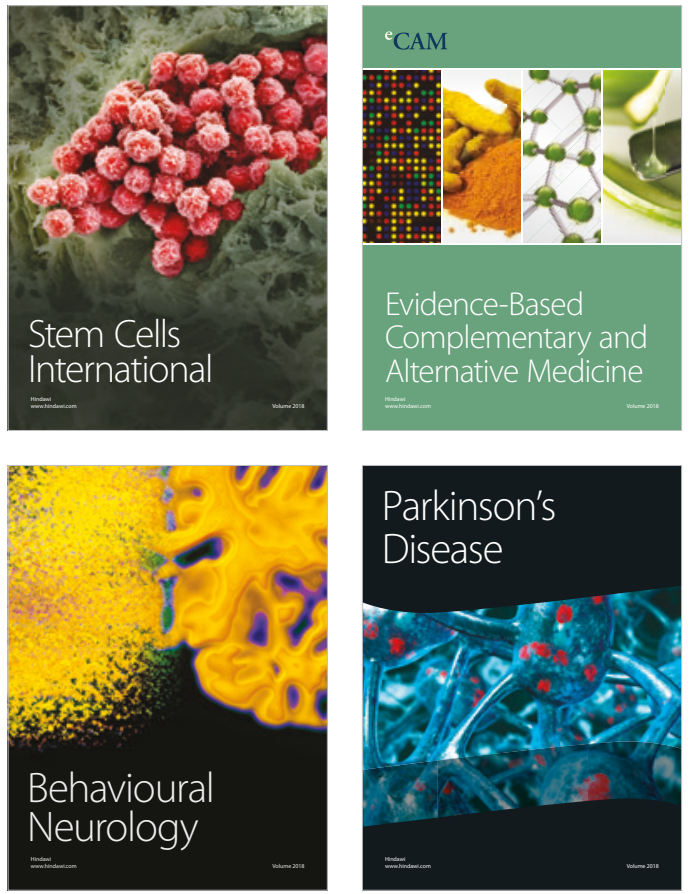

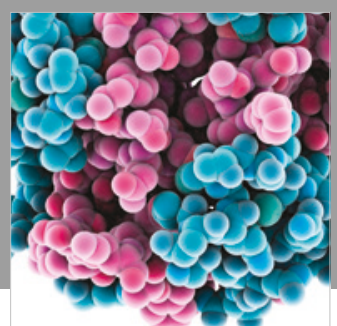

ournal of

Diabetes Research

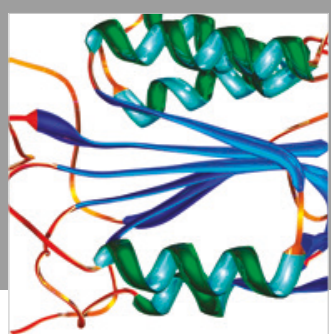

Disease Markers
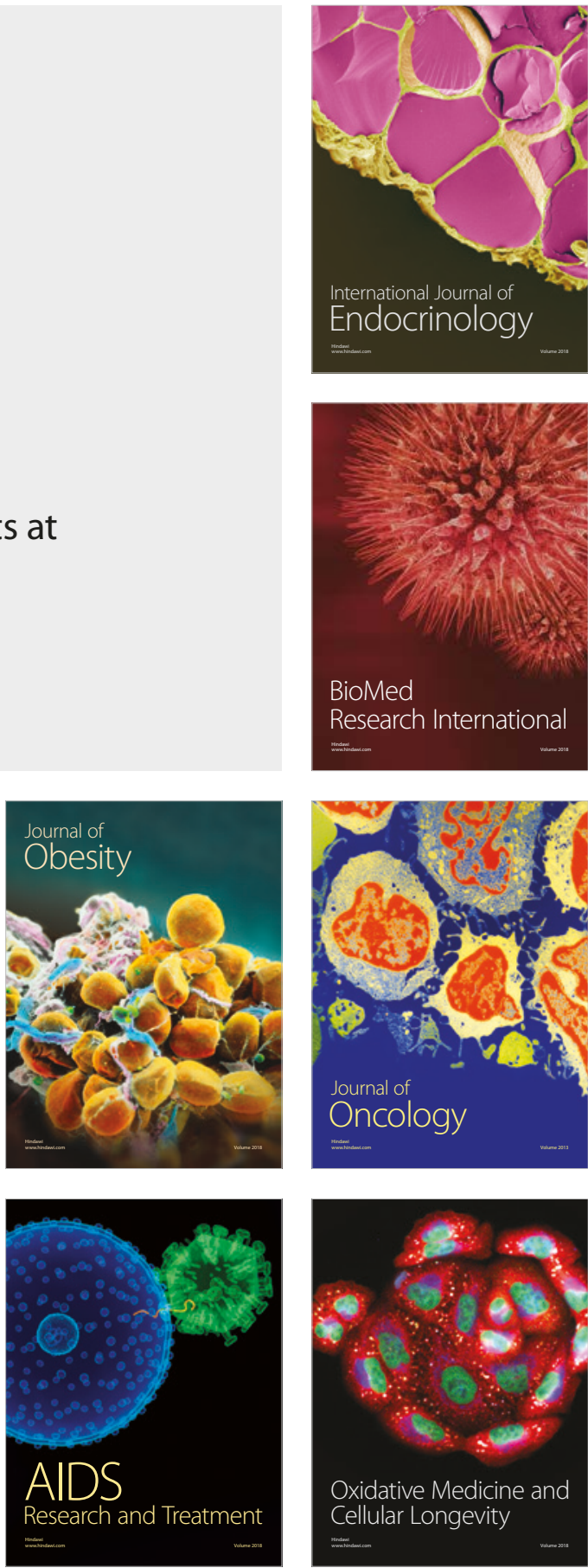